\title{
Relación entre onicofagia y manifestaciones clínicas de trastornos temporomandibulares en dentición mixta primera y/o segunda fase: Una revisión narrativa
}

\author{
Relationship between nail biting and clinical manifestations of \\ temporomandibular disorders in mixed dentition first and/or \\ second phase: A narrative review
}

\author{
Ojeda Léonard $\mathrm{C}^{1}$, Espinoza Rojo $\mathrm{A}^{2}$, Biotti Picand J ${ }^{3}$
}

\begin{abstract}
RESUMEN
Estudios indican que más del $50 \%$ de la población ha padecido alguna vez manifestaciones clínicas relacionadas a trastornos temporomandibulares (TTM). La prevalencia de consultas odontológicas por dolor orofacial ha aumentado, y la edad de inicio de la sintomatología es cada vez más temprana. La etiología de este cuadro es multifactorial, debida entre otros a hábitos parafuncionales. La onicofagia es una parafunción prevalente en niños. Está relacionada a la tensión psíquica, por lo cual el sujeto puede presentar el hábito parafuncional de manera continua o por períodos exacerbados por estrés. Esto justifica su alta prevalencia en la sociedad actual. El objetivo de nuestro estudio fue recopilar de manera clara y actualizada la información disponible y concluir si la literatura científica relaciona la onicofagia y las manifestaciones clínicas de TTM en niños con dentición mixta, primera y/o segunda fase. Gracias a esto surgió la siguiente pregunta clínica: ¿Relaciona la literatura científica actual, el mal hábito de onicofagia con las manifestaciones clínicas de TTM en individuos con dentición mixta primera y/o segunda fase? En cuanto a la metodología utilizada, se procedió al análisis acucioso de cada referencia, dónde se analizó: La calidad de la información, la validez y su consecuente grado de recomendación. Por último, concluimos que para contestar nuestra pregunta clínica y analizar medidas de salud pública, es necesario realizar más estudios sobre el tema. Para lograr generar un impacto a nivel poblacional, es necesario educar y crear consciencia a nivel social, y capacitar más profesionales para tratar estas alteraciones tempranamente.
\end{abstract}

Rev. Clin. Periodoncia Implantol. Rehabil. Oral Vol. 7(1); 37-42, 2014.

Palabras clave: Onicofagia, trastornos témporomandibulares, dentición mixta.

\section{ABSTRACT}

Studies indicate that over $50 \%$ of the population has ever had symptoms related to temporomandibular disorders (TMD). The prevalence of dental visits for orofacial pain has increased and the age of onset of symptoms is increasingly early. The etiology of this condition is multifactorial, due to parafunctional habits, among others. Onychophagia is prevalent in children. It is related to mental stress, so the subject may have continuous parafunctional habits or periods exacerbated by stress. This justifies its high prevalence in modern society. The aim of this study was to compile the available information in the literature to conclude whether onychophagia is related to clinical manifestations of TMD in children with mixed dentition, first and/or second phase. This brought the following clinical question: Does the current literature relate onychophagia with clinical manifestations of TMD in individuals with mixed dentition first and / or second phase? Regarding the methodology, we proceeded to review each reference, where the quality of information, validity and consequent degree of recommendation were analyzed. Finally, we conclude that to answer our question, more clinical studies and public health measures are needed on the subject. To achieve an impact on the population it is necessary to educate and raise awareness at a social level, and train more professionals to treat these disorders early.

Rev. Clin. Periodoncia Implantol. Rehabil. Oral Vol. 7(1); 37-42, 2014.

Key words: Nail biting, temporomandibular disorders, mixed dentition.

\section{INTRODUCCIÓN}

El término "trastorno témporomandibular" es implantado por Welden E. Bell y a lo largo de los años ha recibido distintas denominaciones. Por este motivo, la American Dental Association decide adoptar el concepto unánime de TTM en 1983(1). Corresponde a un término colectivo y heterogéneo que abarca toda alteración funcional del sistema estomatognático (SE). Es un conjunto de signos y síntomas característicos, considerados una subclasificación de los trastornos musculoesqueléticos sistémicos. Presentan una etiología de tipo multifactorial, con una prevalencia variable. De la población adulta entre un 40 y un $75 \%$ presenta al menos un signo y un $33 \%$ al menos un síntoma de TTM. Éstos se manifiestan en los componentes básicos del SE como son: La neuromusculatura, las articulaciones temporomandibulares (ATM), el periodonto y los dientes.

Estos trastornos se caracterizan por ser la principal fuente de dolor orofacial de origen no odontogénico. El concepto de "dolor" presenta un componente biaxial. El eje I representa los factores físicos, es decir, la información somatosensorial y la aparición de impulsos nociceptivos. El eje II representa las alteraciones psicológicas que pueden producir la experiencia dolorosa o bien influir en ella(2).

La onicofagia es el mal hábito de comerse las uñas, es decir, es el resultado de la repetición de una manifestación no deseada que en la frecuencia de la práctica puede instalarse pasando al ámbito inconsciente ${ }^{(3)}$. Es por lo tanto un acto adquirido mediante la experiencia, repetitivo y adverso, proveniente de patrones neuromusculares complejos $^{(4,5,6)}$, dañino para el SE ya que produce un trauma en el sistema masticatorio ${ }^{(4)}$. Además, los malos hábitos son realizados 
regular y automáticamente, sobre los cuáles el sujeto tiene poco control voluntario(6).

Los pacientes que padecen de este mal hábito corresponden tanto a niños como adultos jóvenes ${ }^{(7,8)}$, aunque se ha demostrado que es más prevalente en niñas. "Los niños en particular practican estos hábitos anómalos como una forma de atraer la atención debido a que se encuentran expuestos a un entorno familiar violento, a la falta de atención de los padres, a la falta de madurez emocional, a los cambios constantes en el ambiente familiar, o bien, a modo de imitación"(6).

Las parafunciones orales son comunes ${ }^{(1,9,10,11)}$, y normalmente no dañan el SE. Sin embargo, cuando la actividad excede la tolerancia fisiológica individual, puede causar daño a la dentición, musculatura y ATM $^{(1,9)}$. Numerosos investigadores han propuesto que las parafunciones orales son posibles factores etiológicos para el desarrollo de TTM en un grupo de menor edad, y reportan su asociación con signos y síntomas de disfunción ${ }^{(12-16)}$

La importancia de estos hábitos radica en que "todos los hábitos bucales parafuncionales modifican la posición de los dientes y la relación que guardan éstos entre sí, ya que interfieren con el crecimiento normal y en la función de la musculatura orofacial"(6). "Además, los malos hábitos de posición o funcionamiento de dichos órganos rompen el equilibrio y conducen a disfunciones"(9). Asimismo, se postula que la onicofagia a largo plazo provocaría una disminución del espacio articular, y por ende, desencadenaría una compresión discal ${ }^{(17)}$.

Todo lo mencionado anteriormente, nos permite asociar la onicofagia con un estado de ansiedad y estrés aumentado, lo cual se relaciona a su vez con el eje II de los trastornos dolorosos, dentro de los cuales se encuentran los TTM. "La necesidad de morder e incluso de comerse las uñas está ligada a un estado físico-emocional de ansiedad" $(7,18)$.

J. Pinkham et al. (2005) realizó un estudio en que evaluó la frecuencia de onicofagia según edad(5). Este estudio se realizó entre los 5 y 11 años, con un total de 341 individuos. Se observó que la onicofagia aumenta durante el periodo de dentición mixta entre los 6 y 11 años, y que luego decrece ${ }^{(5,7)}$. La prevalencia aumenta de un $14.2 \%$ hasta un $43.9 \%$ a los 11 años ${ }^{(5)}$.

La presente revisión bibliográfica tiene como objetivo recopilar de manera clara y actualizada la información disponible y concluir si la literatura científica relaciona la onicofagia y las manifestaciones clínicas de TTM en niños con dentición mixta, primera y/o segunda fase, ya que un actuar preventivo mediante actividades de promoción, prevención e intercepción, con intervenciones clínicas oportunas sobre estos factores puede contribuir a disminuir las cifras de afectados ${ }^{(3)}$. En base a esto surge nuestra pregunta clínica: ¿Relaciona la literatura científica actual el mal hábito de onicofagia con las manifestaciones clínicas de TTM en individuos con dentición mixta primera y/o segunda fase?

\section{MATERIALES Y MÉTODO}

Se obtuvo la información online a través de bases de datos como MEDLINE, EMBASE, RHESUS, BIOMED, Índice Médico Español, Excerpa Médica, Trip DataBase, COCHRANE, EBSCO, Lilacs, Pubmed y SciELO. También se incluyó una revisión manual de ciertas revistas científicas tales como $J$ Oral Rehabilitation, European Journal of Orthodontics, European Journal of Paediatric Dentistry, International Journal of Paediatric Dentistry, Journal of Dentistry for Children, Orthodontics and Craniofacial Research, Journal of Orofacial Pain, Journal of Clinical Pediatric Dentistry, The Journal of the American Dental Association, Journal of Dental Research, The Turkish Journal of Pediatrics, Journal of Dentistry of Tehran University of Medical Sciences, The Journal of Craniomandibular Practice, Brazilian Oral Research, Revista CEFAC, Revista Médica Electrónica, Pediatric Nursing, Journal of Applied Behavior Analysis, American Journal of Orthodontics and Dentofacial Orthopedics.

Para realizar la búsqueda se utilizó palabras claves tales como: Temporomandibular disorders, temporomandibular joint (TMJ), craniomandibular disorders, orofacial pain, oral habits, onychophagia, onychophagy, nail biting, children, mixed dentition, parafunctional habits.

Otro recurso utilizado fue la biblioteca de la Universidad de los Andes, José Enrique Diez. Para la selección de la literatura se realizó la búsqueda en base a trastornos temporomandibulares, disfunción orofacial, ortodoncia y odontopediatría.

Todas las búsquedas fueron realizadas desde 1939 hasta el año 2011, y se permitieron los siguientes idiomas: Inglés, español, francés y portugués.

\section{RESULTADOS}

En cuanto a la relación existente entre manifestaciones clínicas de TTM y onicofagia en dentición mixta primera y/o segunda fase, se realizaron distintos estudios en los cuales se concluyó que este mal hábito es la parafunción oral más frecuente en este rango etario. Es importante recalcar que todos estos autores evalúan esta relación en sujetos no pacientes. Farsi (2003)(13), de un total de 1940 individuos detecta que $27.7 \%$ padece onicofagia, resultado similar al obtenido en los estudios de Nilner, Kopp y Wanaman, respectivamente ${ }^{(13)}$. Por otro lado, Merighi et al. $(2007)^{(19)}$, de un total de 79 individuos encuentran que la prevalencia de onicofagia es de un $39.91 \%$. Egermark, Ericsson et al. $(1981)^{(20)}$, encuentran una relación de un $48 \%$ y Widmalm (1995) postula que ésta es mayor al $55 \%{ }^{(13)}$.

Los signos y síntomas evaluados más comúnmente en dentición mixta son: Ruido o salto articular, alteración de la dinámica mandibular, dolor articular y/o muscular espontáneo o a la palpación y cefaleas.

De un total de 82 artículos revisados, tan solo cuatro encuentran una relación positiva entre onicofagia y manifestaciones clínicas de TTM en dentición mixta y tres la descartan (Tablas 1 y 2).

Pereira et al. (2009) ${ }^{(24)}$, postula que la onicofagia es muy común pero sin embargo, no afecta el balance del SE. Por lo tanto, no hay relación significativa entre malos hábitos orales y signos y síntomas de $\operatorname{TTM}^{(24,25)}$. Vanderas $(1995)^{(22)}$, establece que no hay resultados significativos que relacionen onicofagia y signos y síntomas de TTM entre los 6 y 10 años ${ }^{(26)}$. Por último, Alamoudi N (2002) $)^{(27)}$, encuentra que no hay correlación significativa entre onicofagia y signos y síntomas de TTM en niños entre 7 y 14 años de edad ${ }^{(17,22,24,27)}$.

El estudio de Sari S, Sonmez H (2002) y el de Cortese S, Biondi A (2009), postulan que hay una correlación significativa entre onicofagia y signos y síntomas de TTM en dentición mixta ${ }^{(9,21,22)}$ Merighi $L$ (2007), encuentra que hay una relación entre ambos de un $17.72 \%{ }^{(19)}$. Vanderas y Papagiannoulis L (2002), encuentran que los malos hábitos de morder objetos (dentro del cual se incluye la onicofagia) afectaron significativamente la probabilidad de desarrollar alteraciones musculares $^{(23)}$

\section{DISCUSIÓN}

La literatura existente en relación a la pregunta de investigación es poco abundante y mantiene controversia respecto de la importancia de la onicofagia como factor etiológico en la instalación de TTM. Con la finalidad de hacer más ordenada y fluida la discusión, se han analizado diez aspectos distintos, descritos a continuación:

\section{Literatura Científica}

Respecto al tema de investigación, este estudio descubrió que existen pocas comunicaciones al respecto. Además, es posible informar que en la búsqueda realizada entre los años 1939 y 2011, existe gran controversia. Los niveles de evidencia científica encontrados entre los que informan relación corresponden según la clasificación de la North England Evidence Based Guideline Development Project, 1996 de II C (ver Tabla 1). Y en el caso de descartar relación, los niveles de evidencia corresponden a III C (ver Tabla 2).

\section{Pregunta Clínica}

Llama especialmente la atención la gran dispersión en cuanto a definición, terminología y criterios diagnósticos, en relación a TTM y onicofagia, lo que hace más difícil responder a nuestra pregunta clínica.

En virtud de la información discutida y frente a la pregunta clínica expuesta en este estudio, no es posible responder a un paciente con fundamentos científicos que la sola presencia de onicofagia terminará irremediablemente generando manifestaciones de TTM. Sin embargo, una actitud preventiva lleva inevitablemente a disminuir estos hábitos, que en otros aspectos también han causado controversia, respecto de actuar como desencadenantes y/o perpetuantes de alteraciones en el crecimiento orofacial en niños.

Este actuar preventivo debe ser llevado a cabo mediante 
actividades de promoción, prevención e intercepción con intervenciones clínicas oportunas sobre estos factores para disminuir la cifra de afectados.

Además, para lograr contestar nuestra pregunta clínica se sugiere el desarrollo de nuevos estudios al respecto.

\section{Justificación del Estudio}

Relevancia de la investigación de TTM para reconocer el impacto a nivel de salud pública.

"Los TTM son una de las patologías en la sociedad moderna más desafiantes en cuanto a su diagnóstico, pronóstico y tratamiento"(12). "Actualmente, si existe o no relación entre parafunciones orales y TTM es causa de debate" (17).

Los elementos que dificultan el hallazgo de algún tipo de asociación entre malos hábitos y TTM, son las múltiples combinaciones de parafunciones y disfunciones. Además, existen factores variables como son la duración, frecuencia e intensidad del mal hábito, y también un factor clave: La susceptibilidad individual del sistema masticatorio y la capacidad de adaptación de cada individuo ${ }^{(9)}$.

Debemos comprender que para que se lleve a cabo un estudio de TTM en la práctica odontológica, y que éste sea catalogado como problema de salud pública, en primer lugar hay que demostrar que es un problema asociado al SE frecuente en la población actual. Si las manifestaciones clínicas son prevalentes en la población general, los TTM se convierten en un problema importante que debe ser abordado(28). Esto nos habla de la relevancia que pueden llegar a adquirir los TTM si se demuestra su alta prevalencia y alto costo biológico y económico a nivel de la sociedad, convirtiéndose de esta forma en un problema de salud pública.

\section{Definición}

Se observa que en la literatura científica disponible actualmente, hay una confusión entre los distintos términos utilizados para hacer referencia a los TTM, ya que en muchos casos no se utiliza la nómina universal entregada por la American Dental Association en $1983^{(1)}$. Una propuesta interesante es la que hace Sandro Palla en su libro referente a alteraciones "Mioartropatias del sistema masticatorio y dolores orofaciales".

Por otra parte, se comete el error de definir los TTM como una alteración exclusivamente dolorosa y por ende cualquier dolor relacionado a la masticación o a la ATM es diagnosticado, en algunos casos como TTM, y no se considerara la triada necesaria para determinar si un paciente cumple con los criterios diagnósticos de trastornos temporomandibulares, los cuales involucran sintomatología dolorosa y además alteración de la dinámica mandibular(29):

- Dolor temporomandibular localizado en la musculatura mandibular y/o ATM (en reposo o función) ${ }^{(29)}$.

- Dolor a la palpación, ya sea en la ATM o en la musculatura masticatoria(29). - Alteración de la dinámica mandibular ${ }^{(29)}$.

\section{Epidemiología de los TTM}

Para determinar la prevalencia de los TTM, se debe tener claro cuales son los signos y síntomas presentes al momento de realizar el estudio. La exploración clínica demuestra que el $76 \%$ de los sujetos presentan uno o varios signos asociados a TTM. La anamnesis revela que solo $26 \%$ de ellos indica la presencia de un síntoma relacionado con TTM, es decir, $50 \%$ presenta signos que no son referidos como síntomas; estos se denominan signos subclínicos ${ }^{(28)}$.

Un antecedente que llama notoriamente la atención es la alta prevalencia de TTM reportada por los estudios epidemiológicos en la población general y el contraste existente en cuanto al bajo porcentaje de pacientes que consulta por estos trastornos. Seguramente, esto se debe a que los pacientes acuden a la consulta odontológica solamente por dolor, si éste no está presente, se desentienden de los otros síntomas afines. Además, la ignorancia existente en la sociedad sobre la atención dental lleva al paciente a consultar al médico por esta patología estomatológica. Generalmente, los médicos dejan de lado las patologías concernientes a la ATM, por ser ésta un área anatómica muy pequeña y de difícil acceso. Se ha demostrado además, que los TTM pueden no producir síntomas en niños jóvenes, y los casos sintomáticos pueden ser mal diagnosticados como cefaleas u otalgias por el pediatra o el otorrinolaringólogo ${ }^{(30)}$. Lo ideal sería poder realizar un diagnóstico precoz, pero esto se hace imposible por la baja periodicidad de controles odontológicos. Los pacientes, en general, acuden al dentista sólo en caso de dolor y no cada seis meses como es recomendable. Por otro lado, influye también el bajo conocimiento sobre los TTM dentro de la misma comunidad odontológica, dónde se observa que los mismos estomatólogos consideran esta patología como una enfermedad propia de especialistas. En general, se deja de lado la evaluación inicial imprescindible antes de comenzar cualquier tratamiento, para así evitar que el mismo procedimiento odontológico desencadene la alteración. En este caso, la derivación al experto ya sería considerada tardía. Asimismo, el número de especialistas en TTM es bajo, por lo tanto éstos sólo logran abarcar cierto porcentaje de la población con un grado avanzado de la enfermedad, y una vez más, se ve impedida la labor de prevención.

Tabla 1. Nivel de evidencia y grado de recomendación de los artículos científicos que apoyan la relación entre onicofagia y TTM en dentición mixta.

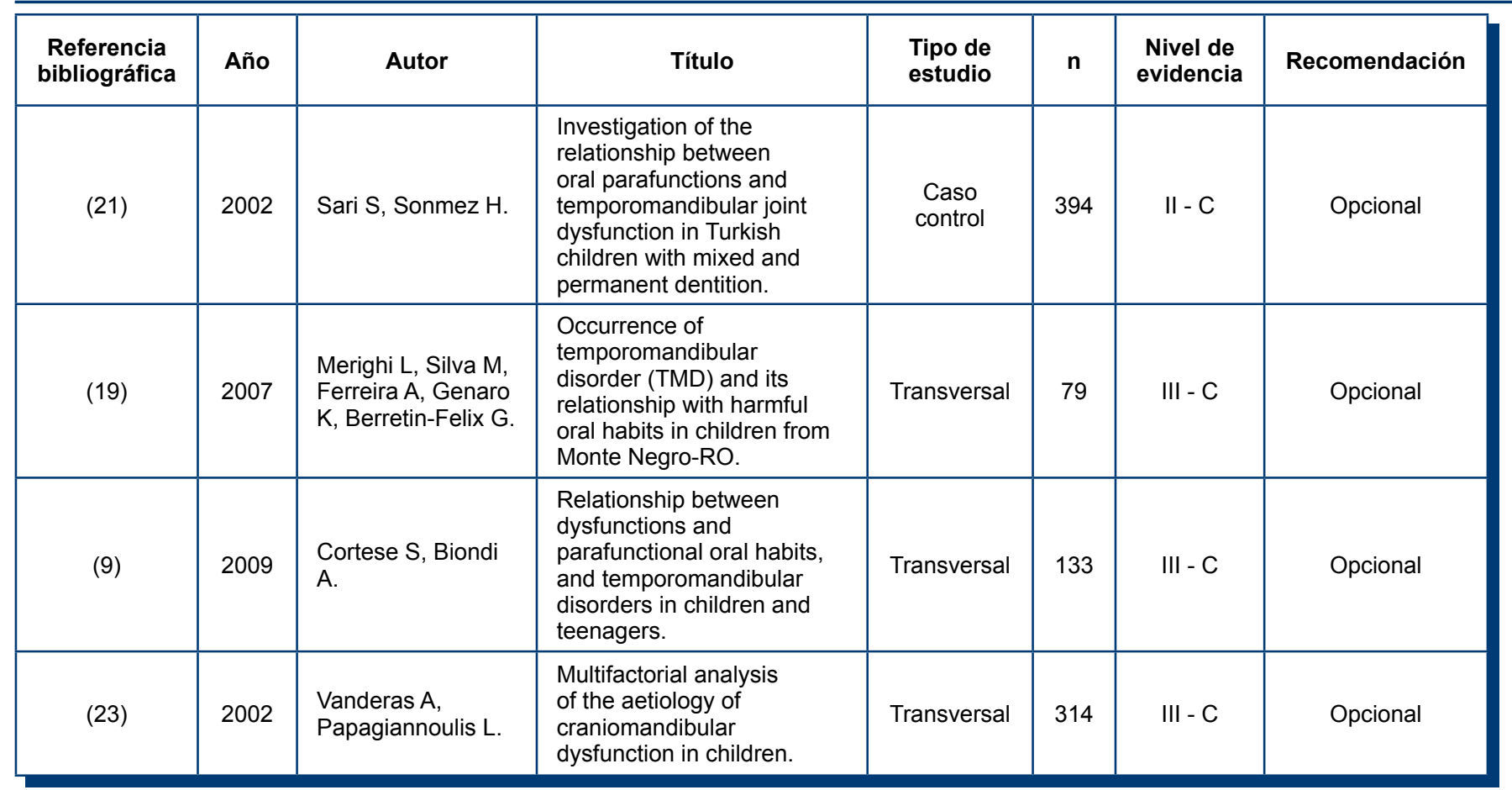


Tabla 2. Nivel de evidencia y grado de recomendación de los artículos científicos que no encuentran relación entre onicofagia y TTM en dentición mixta.

\begin{tabular}{|c|c|l|l|c|c|c|c|}
\hline $\begin{array}{c}\text { Referencia } \\
\text { bibliográfica }\end{array}$ & Año & \multicolumn{1}{|c|}{ Autor } & \multicolumn{1}{|c|}{ Título } & $\begin{array}{c}\text { Tipo de } \\
\text { estudio }\end{array}$ & n & $\begin{array}{c}\text { Nivel de } \\
\text { evidencia }\end{array}$ & Recomendación \\
\hline$(24)$ & 2009 & $\begin{array}{l}\text { Pereira LJ, Costa } \\
\text { RC, França JP, } \\
\text { Pereira SM, Castelo } \\
\text { PM. }\end{array}$ & $\begin{array}{l}\text { Risk indicators for } \\
\text { signs and symptoms } \\
\text { of temporomandibular } \\
\text { dysfunction in children. }\end{array}$ & III-C C & Opcional \\
\hline$(22)$ & 1995 & Vanderas AP. & $\begin{array}{l}\text { Relationship between } \\
\text { craniomandibular } \\
\text { dysfunction and oral } \\
\text { parafunctions in Caucasian } \\
\text { children with and without } \\
\text { unpleasant life events. }\end{array}$ & Transversal & 386 & III - C & Opcional \\
\hline$(27)$ & 2002 & Alamoudi N. & $\begin{array}{l}\text { Correlation between } \\
\text { oral parafunction and } \\
\text { temporomandibular } \\
\text { disorders and emotional } \\
\text { status among Saudi } \\
\text { children. }\end{array}$ & Transversal & 502 & III - C & Opcional \\
\hline
\end{tabular}

Tabla 3. Artículos científicos que consideran el eje Il en el estudio de TTM y modo en que lo evalúan.

\begin{tabular}{|c|c|c|c|c|}
\hline Referencia & Título & Autor & Año & Evaluación del Eje II \\
\hline (9) & $\begin{array}{l}\text { Relationship between dysfunctions } \\
\text { and parafunctional oral habits, and } \\
\text { temporomandibular disorders in } \\
\text { children and teenagers. }\end{array}$ & Cortese SG, Biondi AM. & 2009 & $\begin{array}{l}\text { Compromiso emocional, situaciones } \\
\text { traumáticas, relaciones vinculares, } \\
\text { personalidad, hábitos, nivel de } \\
\text { exigencia, manejo de la agresión, tipo } \\
\text { de expresión verbal y lúdica, grado } \\
\text { de tensión acumulada, manejo de } \\
\text { situaciones difíciles y teoría sobre su } \\
\text { motivo. }\end{array}$ \\
\hline (23) & $\begin{array}{l}\text { Multifactorial analysis of the } \\
\text { aetiology of craniomandibular } \\
\text { dysfunction in children. }\end{array}$ & $\begin{array}{l}\text { Vanderas AP, } \\
\text { Papagiannoulis L. }\end{array}$ & 2002 & $\begin{array}{l}\text { Estrés emocional (miden } \\
\text { catecolaminas urinarias: epinefrina, } \\
\text { norepinefrina y dopamina). }\end{array}$ \\
\hline (34) & $\begin{array}{l}\text { Factors associated with the } \\
\text { temporomandibular disorder, pain } \\
\text { dysfunction syndrome (PDS): } \\
\text { Manchester case-control study. }\end{array}$ & $\begin{array}{l}\text { Macfarlane TV, } \\
\text { Gray RJM, Kincey J, } \\
\text { Worthington HV }\end{array}$ & 2001 & $\begin{array}{l}\text { Cuestionario sobre trastornos del } \\
\text { sueño, cuestionario de salud que } \\
\text { contempla medición de estrés, } \\
\text { cuestionario de enfermedad (mide } \\
\text { adaptación). Los resultados de } \\
\text { los cuestionarios agruparon a los } \\
\text { pacientes hipocondriacos, trastornos } \\
\text { de convicción, percepción somática } \\
\text { vs psicológica de la enfermedad, } \\
\text { inhibición afectiva, disturbios, } \\
\text { afectivos, negación. }\end{array}$ \\
\hline (35) & $\begin{array}{l}\text { Relationship between } \\
\text { craniomandibular dysfunction and } \\
\text { oral parafunctions in Caucasian } \\
\text { children with and without unpleasant } \\
\text { life events. }\end{array}$ & Vanderas AP. & 1995 & $\begin{array}{l}\text { Cuestionario para detectar pacientes } \\
\text { con vidas disconfortables (ansiosos, } \\
\text { nerviosos o con estrés) o pacientes } \\
\text { con vidas confortables (calmos). }\end{array}$ \\
\hline$(27)$ & $\begin{array}{l}\text { Correlation between oral } \\
\text { parafunction and temporomandibular } \\
\text { disorders and emotional status } \\
\text { among Saudi children. }\end{array}$ & Alamoudi N. & 2002 & $\begin{array}{l}\text { Cuestionario que mide estado } \\
\text { emocional. }\end{array}$ \\
\hline
\end{tabular}

\section{Clasificación Diagnóstica}

La clasificación diagnóstica utilizada actualmente para los TTM se basa en la semiología de estos (signos y síntomas). A nuestro parecer, debiera ser cambiada y basada idealmente en la etiología causal de dichos trastornos para lograr una mejor comprensión de estos, y además para facilitar la puesta en marcha de un plan de tratamiento adecuado. En la medida de lo posible, esta clasificación debiera basarse en la sensibilidad y especificidad de los criterios diagnósticos considerados a la fecha( ${ }^{(31,32)}$

Para lograr dichos cambios, es necesario el desarrollo de guías diagnósticas uniformes a nivel de salud pública, y del establecimiento de protocolos de tratamiento. Debemos llamar al tratamiento multidisciplinario, esencial al momento de tratar con este tipo de patología, conformando un equipo de salud para la atención integral del paciente. Además, se debiera trabajar en el desarrollo de medidas preventivas basadas en evidencia científica, la cual en la actualidad es escasa y de bajo nivel.

\section{Etiología}

La frase "etiología multifactorial" ha sido utilizada frecuentemente. Aún no hay consenso en la importancia relativa de factores considerados como etiológicos que promuevan el desarrollo de $\operatorname{TTM}^{(33)}$.

Durante varios años, se ha postulado que los malos hábitos orales serían factores causantes, desencadenantes y/o perpetuantes de los TTM, pero sin embargo la pregunta central a plantearse es: ¿Qué tan involucrado se encuentra cada mal hábito de manera individual (en este caso en particular la onicofagia) en la disfunción del sistema 
masticatorio? Ya que, recordemos, estas entidades al combinarse tienen efecto sinergista. Para la evaluación de la significancia de estos factores en la población, se requieren estudios epidemiológicos, según hábito parafuncional aislado ${ }^{(21)}$.

\section{Eje II}

Otro factor fundamental generalmente dejado de lado a la hora de evaluar trastornos dolorosos, dentro de los cuales se encuentran los TTM, es la gran influencia del eje II. Estas alteraciones son rara vez consideradas en los estudios, y en el caso de ser consideradas, no son evaluadas correctamente. Los estudios que evalúan los factores psicológicos son los siguientes (ver Tabla 3), pero en ningún caso evalúan toda la clasificación diagnóstica de "trastornos mentales", descritas por Okeson ${ }^{(2)}$.

\section{Crítica de los Estudios ya Realizados}

Un gran número de estudios han sido llevados a cabo en distintos países del mundo, para dilucidar la prevalencia de TTM. Los distintos rangos de prevalencia para signos y síntomas de TTM descritos en la literatura científica disponible actualmente se justifican por:

- Diferencias en definición de manifestaciones clínicas de TTM.

- Diferencias poblacionales:

- Metodología para elección de muestra.

- Edades de la muestra.

- Tamaño de muestra.

- Distribución por sexo.

- Influencia hormonal.

- Etnias.

- Diferencias metodológicas:

- Elección de las variables.

- Metodología para la recolección de datos.

- Metodología del examen.

- Uso o no del criterio diagnóstico "RDC/TMD".

- Falta de estandarización de conceptos entre investigadores.

- Número de examinadores involucrados.
- Uso o no de cuestionarios.

- La participación de los responsables de los niños durante la entrevista, etc.

\section{Psicología del Paciente en Etapa Escolar}

Durante el periodo de dentición mixta, "el niño tiene como experiencia central la entrada al colegio ${ }^{(36)}$. Esta experiencia puede ser severa, ya que el periodo escolar trae a la superficie problemas que son el resultado de dificultades previas no resueltas ${ }^{(36)}$. Reacciones como ansiedad, evitación o actitudes negativas pueden ser signos tempranos de dificultades en su ajuste que pueden tornarse en problemas a futuro"(36). La onicofagia es un mal hábito estrechamente relacionado con estas manifestaciones psicológicas, por lo cuál podemos entender su alta prevalencia en este tipo de individuos. Esto explicaría bajo nuestro punto de vista, la alta prevalencia de onicofagia en dentición mixta, pero para comprobarlo merece ser analizado en un estudio bien diseñado.

Por último, y a modo de conclusiones, para lograr contestar nuestra pregunta clínica y analizar medidas aplicables en salud pública basadas en la evidencia científica, es necesario el desarrollo de estudios bien diseñados, de tipo longitudinal, actualizados, con criterios definidos previamente y que sean unánimes a nivel mundial para lograr hablar un lenguaje en común respecto a los TTM y a la onicofagia en particular. Con esto nos referimos a la creación de protocolos diagnósticos nacionales e internacionales, con operadores calibrados, según normas propuestas por la OMS. Además, para lograr generar un impacto a nivel poblacional, es necesario educar y crear consciencia a nivel social sobre la importancia de la salud oral y la gran relevancia de la prevención. Sumado a esto, se necesita tener más profesionales capacitados para tratar estas alteraciones, o en el último de los casos, que sean capaces de detectarlos tempranamente y derivar a tiempo en vez de perpetuarlos con los mismos tratamientos odontológicos.

\section{CONFLICTO DE INTERESES} ningún tipo.
Los autores declaran no presentar conflictos de interés de

\section{REFERENCIAS BIBLIOGRÁFICAS}

1. Okeson JP. Tratamiento de oclusión y afecciones temporomandibulares. Elsevier España; 2008: p. 130-224

2. Okeson JP. Dolor orofacial según Bell. Quintessence; 2007.

3. Martínez I, Toledo T, Prendes A, Carvajal T, Delgado A, Morales J. Factores de riesgo en pacientes con disfunción temporomandibular. Rev Méd Elec, 2009; 31(4). Extraída de: http://www.revmatanzas.sld.cu/revista\%20medica/ano\%202009/vol4\%202009/tema04. $\mathrm{htm}$; sin número de páginas.

4. Seraj B, Ahmadi R, Mirkarimi M, Ghadimi S, Beheshti M. Temporomandibular disorders and parafunctional habits in children and adolescence: A review. Tehran Univ Med Dent J, 2009; 6(1): 37-45.

5. Pinkham JR. Pediatric dentistry: Infancy through adolescence. Casamassimo P et al, editors. Philadelphia: Elsevier Saunders; c2005.

6. Murrieta-Pruneda JF, Allendelagua B, Pérez S, Juárez-López LA, Linares $V$, Meléndez $\mathrm{O}$ et al. Prevalencia de hábitos bucales parafuncionales en niños de edad preescolar en ciudad Nezahualcóyotl, Estado de México, 2009. Bol Med Hosp Infant Mex, 2011; 68(1): 26-33.

7. Tanaka OM, Vitral RWF, Tanaka GY, Guerrero AP, Camargo ES. Nailbiting, or onychophagia: A special habit. AJO-DO, 2008; 134(2): 305-308.

8. Birch L. The incidence of nail biting among school children. Brit J Educ Psychol, 1955; 25(2): 123-128.

9. Cortese SG, Biondi AM. Relationship between dysfunctions and parafunctional oral habits, and temporomandibular disorders in children and teenagers. Arch Argent Pediatr, 2009; 107: 134-138.

10. Pereira LJ, Pereira-Cenci T, Cury AADB, Pereira SM, Pereira AC, Ambosano GMB et al. Risk indicators of temporomandibular disorder incidences in early adolescence. Pediatr Dent, 2010; 32(4): 324-328.

11. Nowak AJ, Warren JJ. Infant oral health and oral habits. Pediatr Clin N Am, 2000; 47(5): 1043-1066.

12. Jerolimov V. Temporomandibular disorders and orofacial pain. J Med Scien, 2009; 33: 53-77

13. Farsi N. Symptoms and signs of temporomandibular disorders and oral parafunctions among Saudi children. J Oral Rehabil, 2003; 30(12): 1200-1208. 14. Tosato J, Biasotto-Gonzalez D. Symptomatology of the temporomandibular dysfunction related to parafunctional habits in children. Braz J Oral Sci, 2005; 14(4): 787-790.
15. Gavish A, Halachmi M, Winocur E, Gazit E. Oral habits and their association with signs and symptoms of temporomandibular disorders in adolescent girls. J Oral Rehabil, 2000; 27(1): 22-32.

16. Teixeira ACB, Marcucci G, Luz JGC. Prevalência das maloclusões e dos índices anamnésicos e clínicos, em pacientes com disfunção da articulação temporomandibular. Rev Odontol Univ São Paulo, 1999; 13(3): 251-256.

17. Michelotti A, Cioffi I, Festa P, Scala G, Farella M. Oral parafunctions as risk factors for diagnostic TMD subgroups. J Oral Rehabil, 2010; 37(3): 157-162.

18. Massler M, Malone AJ. Nailbiting-A review. J Pediatr, 1950; 36(4): 523-531.

19. Merighi LBM, Silva MMA, Ferreira AT, Genaro KF, Berretin-Felix G. Occurrence of temporomandibular disorder (TMD) and its relationship with harmful oral habits in children from Monte Negro-RO. Revista CEFAC, 2007; 9(4): 497-503.

20. Egermark-Eriksson I, Carlsson GE, Ingervall B. Prevalence of mandibular dysfunction and orofacial parafunction in 7-, 11-and 15-year-old Swedish children. Eur J Orthod, 1981; 3(3): 163-172.

21. Sari S, Sonmez H. Investigation of the relationship between oral parafunctions and temporomandibular joint dysfunction in Turkish children with mixed and permanent dentition. J Oral Rehabil, 2002; 29(1): 108-112.

22. Castelo P, Gavião M, Pereira L, Bonjardim L. Relationship between oral parafunctional/nutritive sucking habits and temporomandibular joint dysfunction in primary dentition. Int J Paediatr Dent, 2005; 15(1): 29-36.

23. Vanderas A, Papagiannoulis L. Multifactorial analysis of the aetiology of craniomandibular dysfunction in children. Int J Paediatr Dent, 2002; 12(5): 336-346.

24. Pereira LJ, Costa RC, França JP, Pereira SM, Castelo PM. Risk indicators for signs and symptoms of temporomandibular dysfunction in children. $J$ Clin Pediatr Dent, 2009; 34(1): 81-86.

25. Winocur E, Gavish A, Finkelshtein T, Halachmi M, Gazit E. Oral habits among adolescent girls and their association with symptoms of temporomandibular disorders. J Oral Rehabil, 2001; 28(7): 624-629.

26. Olsson M, Lindqvist B. Mandibular function before orthodontic treatment. Eur J Orthod, 1992; 14(1): 61.

27. Alamoudi N. Correlation between oral parafunction and temporomandibular disorders and emotional status among Saudi children. J Clin Pediatr Dent, 2002 26(1): 71-80. 
28. Kharbanda O, Sidhu S, Sundaram K, Shukla D. Oral habits in school going children of Delhi: A prevalence study. J Indian Soc Pedo Prev Dent, 2003; 21(3): 120-124.

29. Manns A, Biotti J, Rocabado M. Trastornos o desórdenes temporomandibulares. [De próxima aparición] Chile; 2008.

30. Muhtaroğulları M, Demirel F, Saygılı G. Temporomandibular disorders in Turkish children with mixed and primary dentition: Prevalence of signs and symptoms. Turk $J$ Pediatr, 2004; 46(2): 159-163.

31. International RDC-TMD Consortium Network > Home [Internet]. [cited 2012 Jan

13]. Available from: http://www.rdc-tmdinternational.org/Home.aspx

32. International RDC-TMD consortium. Multi-site RDC/TMD Validation Study.

Specifications for TMD. Examination Gold Standard Examine. 10 Feb 2005.

33. Mohlin B, Pilley J, Shaw W. A survey of craniomandibular disorders in 1000

12-year-olds. Study design and baseline data in a follow-up study. Eur J Orthod

1991; 13(2): 111-123.
34. Macfarlane T, Gray R, Kincey J, Worthington H. Factors associated with the temporomandibular disorder, pain dysfunction syndrome (PDS): Manchester casecontrol study. Oral Dis, 2001; 7(6): 321-330.

35. Vanderas A. Relationship between craniomandibular dysfunction and oral parafunctions in Caucasian children with and without unpleasant life events. J Oral Rehabil, 1995; 22(4): 289-294.

36. Guerrero A. Desarrollo del niño durante el periodo escolar [Monografía en Internet]. Chile. Manual de Pediatría, Pontificia Universidad Católica de Chile [Acceso el 3 de octubre 2012]. Disponible en: http://escuela.med.puc.cl/paginas/ publicaciones/manualped/DessPsicEsc. 